\title{
Aplicabilidade da termografia infravermelha na odontologia: Uma revisão integrativa
}

\author{
Applicability of infrared thermography in dentistry: An integrative review \\ Aplicabilidad de la termografía infrarroja en odontología: Una revisión integradora
}

Recebido: 22/06/2021 | Revisado: 28/06/2021 | Aceito: 02/07/2021 | Publicado: 15/07/2021

\author{
Karolyne Pereira da Silva Cavalcanti \\ ORCID: https://orcid.org/0000-0003-3959-7089 \\ Faculdade Nova Esperança, Brasil \\ E-mail: kpscazevedo@gmail.com \\ Herrison Félix Valeriano da Silva \\ ORCID: https://orcid.org/0000-0001-6714-3151 \\ Universidade Federal da Paraíba, Brasil \\ E-mail: Herrison.felix.vds@gmail.com \\ Fernanda Clotilde Mariz Suassuna \\ ORCID: https://orcid.org/0000-0001-5846-288X \\ Faculdade Nova Esperança, Brasil \\ E-mail: fernandacosta3@hotmail.com \\ Renally Bezerra Wanderley e Lima \\ ORCID: https://orcid.org/0000-0003-4477-7850 \\ Faculdade Nova Esperança, Brasil \\ E-mail: fernandacosta3@hotmail.com \\ Thauany Vasconcelos Soares da Silva \\ ORCID: https://orcid.org/0000-0002-6831-9224 \\ Universidade Federal da Paraíba, Brasil \\ E-mail: vasconcelosthauany@gmail.com \\ Manoel Pereira de Lima \\ ORCID: https://orcid.org/0000-0002-5605-0651 \\ Universidade Federal da Paraíba, Brasil \\ E-mail: Manoel_lima18@yahoo.com.br \\ Maria Eduarda de Araujo Cruz \\ ORCID: https://orcid.org/0000-0001-8876-8249 \\ Faculdade Nova Esperança, Brasil \\ E-mail: eduardaaraujo207@gmail.com \\ Stéfani Caroline Ferriolli \\ ORCID: https://orcid.org/0000-0003-0019-6026 \\ Universidade Estadual Paulista, Brasil \\ E-mail: stefani.ferriolli@outlook.com \\ Jussara da Silva Barbosa \\ ORCID: https://orcid.org/0000-0001-6123-5266 \\ Faculdade Nova Esperança, Brasil \\ E-mail: barbosajsara@gmail.com
}

\begin{abstract}
Resumo
Este estudo teve como objetivo realizar uma revisão integrativa para analisar a aplicabilidade da termografia infravermelha na Odontologia. Para a busca, nas bases de dados Scielo e Pubmed, foram utilizadas as seguintes palavras-chave: "Thermography"; "Body Temperature"; "Microcirculation"; "Dentistry"; "Oral”, utilizando o operador booleano "AND”. Foram incluídos nesta revisão, estudos que utilizaram a termografia no acompanhamento de terapias, bem como estudos clínicos e laboratoriais, sendo excluídos os estudos não relacionados ao objetivo desta pesquisa, estudos realizados em período maior do que cinco anos, trabalhos de conclusão de curso, dissertações, trabalhos publicados em duplicatas, anais e cartas ao editor. Desta maneira, foram encontrados 140 artigos. Após a triagem por títulos e resumos, um total de 117 foram excluídos. Ao final foram incluídos 15 estudos nesta revisão. Com base na pesquisa realizada, verifica-se que ainda não existe uma quantidade significativa de artigos que abordem as aplicações da termografia na Odontologia, havendo ainda diversas áreas que podem utilizá-la como ferramenta de diagnóstico e investigação eficaz.
\end{abstract}

Palavras-chave: Termografia; Temperatura corporal; Microcirculação; Odontologia; Oral.

\section{Abstract}

This study aimed to carry out an integrative review to analyze the applicability of infrared thermography in Dentistry. For the search, in the Scielo and Pubmed databases, the following keywords were used: "Thermography"; "Body 
Temperature"; "Microcirculation"; "Dentistry"; "Oral", using the Boolean operator "AND”. Included in this review were studies that used thermography in the follow-up of therapies, as well as clinical and laboratory studies, excluding studies not related to the objective of this research, studies conducted over a period of more than five years, course completion papers, dissertations, works published in duplicates, annals and letters to the editor. Accordingly, 140 articles were found. After screening by titles and abstracts, a total of 117 were excluded. At the end, 15 studies were included in this review. Based on the research carried out, it appears that there is still no significant number of articles that address the applications of thermography in Dentistry, and there are still several areas that can use it as an effective diagnostic and investigation tool.

Keywords: Thermography; Body temperature; Microcirculation; Dentistry; Oral.

\section{Resumen}

Este estudio tuvo como objetivo realizar una revisión integradora para analizar la aplicabilidad de la termografía infrarroja en Odontología. Para la búsqueda, en las bases de datos Scielo y Pubmed, se utilizaron las siguientes palabras clave: "Termografía"; "Temperatura corporal"; "Microcirculación"; "Odontología"; "Oral", utilizando el operador booleano "Y". Esta revisión incluyó estudios que utilizaron termografía en el seguimiento de terapias, así como estudios clínicos y de laboratorio, excluyendo estudios no relacionados con el objetivo de esta investigación, estudios realizados durante un período superior a cinco años, trabajos de finalización de cursos, disertaciones. trabajos publicados en duplicados, anales y cartas al editor. Así, se encontraron 140 artículos. Después de la selección de títulos y resúmenes, se excluyó un total de 117. Al final, se incluyeron 15 estudios en esta revisión. Con base en la investigación realizada, parece que aún no existe una cantidad significativa de artículos que aborden las aplicaciones de la termografía en Odontología, y aún existen várias áreas que pueden utilizarla como una herramienta eficaz de diagnóstico e investigación.

Palabras clave: Termografía; Temperatura corporal; Microcirculación; Odontología; Oral.

\section{Introdução}

A temperatura humana é um sinal vital que pode ser aferido em um único ponto ou em diversos pontos cutâneos de forma a controlar a termorregulação e as doenças a ela relacionadas. O corpo humano aquece e se resfria continuamente em busca de um estado de equilíbrio térmico. Assim como a temperatura central, a temperatura da superfície cutânea também é um parâmetro fisiológico importante que pode ser monitorado para o controle de patologias (Brioschi et al.,2010).

O primeiro relato de diagnóstico termobiológico foi descoberto nos registros de Hipócrates, onde observou que ao espalhar lama sobre uma determinada região do corpo do paciente esta área secava primeiro, indicando assim uma possível doença orgânica subjacente. Segundo Hipócrates, se existir a presença excessiva de calor ou frio em alguma região do corpo, uma patologia poderá estar presente (Brioschi et al.,2001; Brioschi et al.,2003).

A Termografia Infravermelha é um exame de imagem complementar ainda emergente para diagnóstico, monitoramento terapêutico e prognóstico na medicina, tal exame consiste na utilização de uma câmera térmica infravermelha para detecção do calor emitido pela radiância do corpo humano em análise, na faixa do infravermelho longo (Brioschi,2011). Em 1956, a termografia infravermelha foi introduzida pela primeira vez por R. N. Lawson para auxiliar no diagnóstico de câncer de mama, e apenas em 1987, foi reconhecida como uma ferramenta de diagnóstico viável, mostrando-se uma grande aliada para o diagnóstico clínico (Brioschi et al.,2010; Brioschi,2011; Amorim et al.,2018).

$\mathrm{O}$ uso da termografia pode ser útil para um correto diagnóstico, sendo utilizado como um exame complementar ao exame físico, auxiliando significativamente também no acompanhamento de terapias e podendo ser realizado em qualquer faixa etária (Brioschi,2011). É potencialmente indicado para documentar doenças de tecidos moles, sendo evidenciada também sua importância na medicina do esporte, doenças ligadas a dores musculares como mialgias, bem como doenças reumáticas como artrose, apresentando uma visualização anatômica das estruturas, sendo possível analisar os aspectos fisiológicos da região e é realizada em um ambiente com temperatura controlada (Brioschi,2011; Barbosa et al.,2020).

Além dos exemplos que se destacam na medicina, a termografia, tem amplo potencial em aplicações na odontologia sendo uma técnica bastante promissora na área de Endodontia (Behnia et al.,2001), Estomatologia (Marroquín et al.,2015), Patologia (Iosif et al.,2016) Implantodontia (Chakrabort et al,2016), Cirurgia Oral (Harder et al.,2018) , Disfunção 
Temporomandibular (Christensen et al.,2012), podendo também ser aplicada em estudos para análise da dor orofacial e para rastreamento de febre por meio de uma investigação facial (Haddad et al.,2012).

Predominam hoje métodos não invasivos e uma visão holística em todas as áreas da saúde. A termografia por se tratar de um método de imagem não invasivo, que mede e mapeia a distribuição de temperatura emitida pela superfície do corpo, não implicando na utilização de radiação ionizante, acesso venoso, contrastes radiológicos ou quaisquer outros procedimentos invasivos, sendo um método simples, seguro, rápido, de baixo custo e não provocando nenhum dano ou desconforto ao paciente, mostra seu potencial futuro, como exame importante para o tratamento e investigação de patologias diversas e na análise comportamental em laboratório, e então surge a necessidade de entrarmos em profundidade no tema (Brioschi et al.,2010).

Diante disso, este estudo teve como objetivo realizar uma revisão integrativa para analisar a aplicabilidade da termografia infravermelha na Odontologia bem como entender os parâmetros utilizados em diferentes estudos para adequação dos exames termográficos.

\section{Metodologia}

O presente estudo tratou-se de uma revisão integrativa da literatura científica de caráter qualitativo (Koche,2011). Foi realizado um levantamento bibliográfico sobre a aplicabilidade da Termografia infravermelha na Odontologia, realizando uma síntese sobre os resultados encontrados na prática acerca do tema, baseando-se em evidências científicas.

As seguintes etapas metodológicas foram seguidas para a realização desta pesquisa:

- Levantamento Bibliográfico: realizado nas bases de dados SciELO e Pubmed;

- Cruzamento das Palavras-chaves: "Thermography"; "Body Temperature"; "Microcirculation"; "Dentistry"; "Oral", utilizando o operador booleano "AND", formando uma chave de busca.

- Leitura dos títulos e resumos: nesta fase foram selecionados os principais estudos de acordo com o objetivo ora proposto;

- Leitura dos artigos na íntegra: após este processo foi construída uma tabela, com as informações levantadas dos estudos para compor os resultados desta revisão.

Para esta pesquisa foram utilizados como critério de inclusão estudos realizados nos últimos 5 anos (2016 a 2021), publicados na íntegra nos idiomas inglês, português e espanhol, bem como, estudo laboratoriais e ensaios clínicos que utilizaram a Termografia infravermelha em sua metodologia. Foram excluídos os estudos que não estavam de acordo com o objetivo da presente pesquisa, como também, dissertações, teses e trabalhos de conclusão de curso.

Para análise dos dados extraídos foi realizada uma síntese qualitativa e detalhada dos resultados dos estudos incluídos.

Após o cruzamento das palavras-chaves nas bases de dados foram encontrados 140 artigos. Após a triagem por títulos e resumos, 117 artigos foram excluídos. Destes, 23 artigos foram selecionados para leitura do texto na íntegra, dos quais 8 foram excluídos, conforme descrito na Figura 1. 
Figura 1. Fluxograma com processo para seleção dos artigos incluídos para esta revisão.

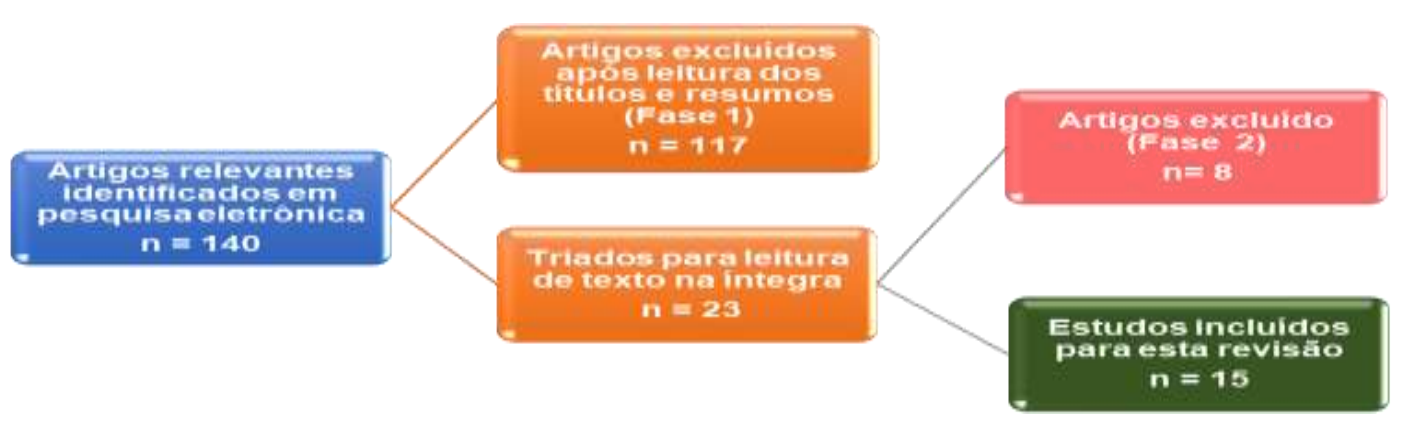

Fonte: Autores.

\section{Resultados e Discussão}

Tabela 1. Áreas, ano de publicação, autor, tipo de estudo, número de pacientes, objetivos e resultados dos artigos analisados no estudo. $\mathrm{n}=15$.

\begin{tabular}{|c|c|c|c|c|c|c|}
\hline ÁREAS & ANO & AUTOR & $\begin{array}{l}\text { TIPO DE } \\
\text { ESTUDO }\end{array}$ & $\begin{array}{c}\text { NÚMERO } \\
\text { DE } \\
\text { PACIENTES }\end{array}$ & OBJETIVOS & RESULTADOS \\
\hline Cirurgia & 2019 & Endo et al. & $\begin{array}{l}\text { Ensaio } \\
\text { Clínico }\end{array}$ & $\mathrm{n}=10$ & $\begin{array}{l}\text { Avaliar a utilidade da } \\
\text { termografia para quantificar } \\
\text { a temperatura facial antes e } \\
\text { após a cirurgia ortognática e } \\
\text { fixação intermaxilar, e os } \\
\text { efeitos desses } \\
\text { procedimentos ortognáticos } \\
\text { na temperatura facial. }\end{array}$ & $\begin{array}{l}\text { A termografia foi útil para } \\
\text { avaliação quantitativa da } \\
\text { temperatura facial em } \\
\text { pacientes submetidos à } \\
\text { cirurgia ortognática. As } \\
\text { mudanças na temperatura } \\
\text { facial foram devidas } \\
\text { predominantemente à } \\
\text { inflamação após a cirurgia. }\end{array}$ \\
\hline $\begin{array}{l}\text { Temperatura } \\
\text { facial }\end{array}$ & 2020 & $\begin{array}{l}\text { Scarano, } \\
\text { Inchingolo, } \\
\text { Lorusso }\end{array}$ & $\begin{array}{l}\text { Ensaio } \\
\text { Clínico }\end{array}$ & $\mathrm{n}=20$ & $\begin{array}{l}\text { Comparar a temperatura da } \\
\text { pele facial e o fluxo de calor } \\
\text { ao usar máscaras médico- } \\
\text { cirúrgicas e respiradores } \\
\text { N95. }\end{array}$ & $\begin{array}{c}\text { Uma diferença } \\
\text { estatisticamente significativa } \\
\text { na umidade, calor, } \\
\text { dificuldade respiratória e } \\
\text { desconforto estiveram } \\
\text { presentes entre os grupos. Os } \\
\text { resultados da Termografia } \\
\text { sugerem que o os } \\
\text { respiradores N95 são capazes } \\
\text { de induzir um aumento da } \\
\text { temperatura da pele facial, } \\
\text { maior desconforto e menor } \\
\text { adesão ao uso quando } \\
\text { comparada às máscaras } \\
\text { médico-cirúrgicas. }\end{array}$ \\
\hline Dor Miofascial & 2019 & $\begin{array}{l}\text { Altindiş e } \\
\text { Güngörmş }\end{array}$ & $\begin{array}{l}\text { Ensaio } \\
\text { Clínico }\end{array}$ & $n=20$ & $\begin{array}{l}\text { Comparar e avaliar os } \\
\text { efeitos da placa oclusal e do } \\
\text { laser de baixa intensidade } \\
\text { clínica e termograficamente } \\
\text { em pacientes com síndrome } \\
\text { de dor miofascial (MPS). }\end{array}$ & $\begin{array}{l}\text { A terapia com placa oclusal e } \\
\text { a terapia com laser de baixa } \\
\text { intensidade foram eficazes no } \\
\text { tratamento de MPS, e quando } \\
\text { os dados termográficos foram } \\
\text { levados em consideração, os } \\
\text { tratamentos LLLT poderiam } \\
\text { fornecer mais resultados } \\
\text { vantajosos nestes pacientes. }\end{array}$ \\
\hline
\end{tabular}


Dor Orofacial
Rytivaara et al.

Ensaio

Clínico

$n=40$
Avaliar como os achados da termografia relacionam a dor aos sintomas e sinais de disfunção

temporomandibular (DTM).

A termografia pode ser uma ferramenta potencial no diagnóstico de pacientes com

DTM no sexo feminino. As informações térmicas avaliadas em áreas faciais específicas podem ajudar a discriminar pacientes com DTM de pacientes sem DTM e pode ser usada para quantificar a dor associada a DTM.

Revisar sistematicamente a eficácia científica da termografia infravermelha no diagnóstico de distúrbios da articulação temporomandibular (DTM).

Avaliar pacientes com e sem DTM muscular por meio da termografia infravermelha de acordo com as diferenças de radiância térmica por meio de testes quantitativos de sensibilidade e especificidade; e avaliar a assimetria térmica e a correlação da intensidade térmica com a intensidade da dor à palpação.

Descrever as etapas necessárias e elementares no diagnóstico e tratamento de um instrumentista de sopro com disfunção temporomandibular, com a introdução da termografia infravermelha neste procedimento.
A literatura ainda é escassa quanto à confiabilidade da termografia infravemelha para o diagnóstico de DTM.
A termografia infravermelha resultou em baixa acurácia, dificultando a diferenciação da DTM por meio da análise termográfica. A intensidade da dor à palpação em pacientes com DTM pode ser acompanhada por uma diminuição da temperatura local de acordo com os parâmetros térmicos.
DTM

2018 Clemente et al. $\begin{gathered}\text { Estudo de } \\ \text { Caso }\end{gathered}$

Diegritz et al. In vitro $\mathrm{n}=45$
Determinar o aumento da temperatura na superfície radicular durante a compactação vertical a quente por meio de três diferentes sistemas de obturação.
Com exames termográficos infravermelhos, bem como o uso de sensores nas análises, uma eventual assimetria nas

ATMs foi encontrada. $\mathrm{O}$ emprego dessas técnicas na odontologia criará a chance de prevenir o uso excessivo de algumas estruturas anatômicas, com o

diagnóstico precoce e o correto monitoramento dessa área.

O aumento da temperatura na superfície radicular durante a aplicação da compactação vertical apresenta considerável variabilidade dependendo do sistema de obturação, de acordo com os dados termográficos.

Os exercícios de alongamento podem ser vistos como medidas preventivas eficazes para evitar estiramentos musculares causados por posturas de trabalho errôneas, no entanto, mais estudos com termografia e outros métodos são necessários para se chegar a uma conclusão definitiva. 


\begin{tabular}{|c|c|c|c|c|}
\hline 2016 & $\begin{array}{c}\text { Möhlhenrich et } \\
\text { al. }\end{array}$ & In vitro & $\mathrm{n}=10$ & $\begin{array}{l}\text { Determinar como a } \\
\text { densidade óssea afeta a } \\
\text { temperatura no osso } \\
\text { artificial e na broca. }\end{array}$ \\
\hline
\end{tabular}

Implantodontia
O desenvolvimento térmico depende da densidade óssea, com o aumento da densidade causando um aumento da temperatura. No entanto, este efeito é reduzido com o aumento do diâmetro da broca. Isso pode ser importante no que diz respeito às reações ósseas e também em termos de desgaste da ferramenta.

O material da broca Medir as mudanças de temperatura durante osteotomias simuladas em osso de costela bovina, in vitro.

influência nas mudanças de temperatura, de acordo com os parâmetros termográficos, que ocorrem em sua porção apical durante o preparo ósseo para a colocação do implante.

A perfuração simples pode gerar mais calor do que a perfuração sequencial tradicional, e a densidade óssea, bem como o diâmetro da broca, influenciam os aumentos térmicos.

Particularmente no osso de baixa densidade, a perfuração sequencial convencional parece aumentar menos a temperatura.

A distribuição da temperatura facial demonstrou várias tendências com o tempo em diferentes condições. Os tipos de máscara facial tiveram efeitos significativos na temperatura facial e no conforto térmico percebido

Medir com termografia infravermelha as mudanças de temperatura na superfície adjacente do teto da câmara de dentes pré-molares extraídos de pacientes jovens e velhos. propriedades isolantes
A dentina teve um efeito significativo em limitar o aumento da temperatura gerado durante a polimerização de materiais provisórios, indicando boas térmicas deste tecido.

Procedimentos apropriados de resfriamento, segundo os dados termográficos, e brocas finas de carboneto de tungstênio devem ser usados durante a remoção dos adesivos remanescentes após a descolagem do bráquete para prevenir reações pulpares adversas.

Fonte: Autores.

Os estudos selecionados nesta revisão trazem como principal função do exame termográfico a identificação de variações de temperatura em tecidos moles que não podem ser evidenciadas por exames laboratoriais, radiográficos ou eletroneuromiográficos, sendo possível analisar os aspectos fisiológicos da região, destacando-se como método complementar 
ao diagnóstico, auxiliando na identificação de patologias. Sendo uma técnica bastante promissora em diversas áreas, a termografia, por ser um método rápido, não invasivo, de baixo custo, e complementar, pode ser indicada antes, durante e após o tratamento (Christensen et al.,2012).

Nos casos analisados, foi evidenciada que para realização dos exames termográficos é de grande importância a tomada de medidas e protocolos no âmbito a ser realizada a obtenção das imagens, a fim de minimizar as interferências que podem gerar inconsistência nos resultados obtidos no exame.

Há um consenso entre os artigos de que o exame termográfico necessita de um preparo para a sua realização, onde todos os voluntários recebem instruções no momento da triagem, a fim de evitar que fatores externos interfiram na aquisição da imagem, como: exercícios físicos, não utilizar nenhuma fonte de calor, maquiagem ou loção no rosto, não tomar analgésicos, anti-inflamatórios ou corticoides e não coçar, esfregar ou arranhar a pele (Brioschi et al.,2010).

Também considerados como parâmetros ideais para assegurar resultados compatíveis com a realidade, os termogramas são comumente obtidos em sala com iluminação fluorescente artificial, equipada com ar-condicionado, mantendo-se a temperatura ambiente em $22 \pm 1,0^{\circ} \mathrm{C}$ e umidade relativa do ar entre $40 \%$ e $60 \%$, verificado por um termohigrômetro digital, colocado próximo ao paciente durante a realização do exame. Para a captura da imagem termográfica uma câmera infravermelha é utilizada e ligada de 15 a 20 minutos antes do início da coleta para estabilizar a temperatura, é necessário também que o paciente permaneça durante 20 minutos parado na sala de espera para estabilizar a temperatura (Brioschi et al.,2010; Christensen et al.,2012).

$\mathrm{O}$ ambiente clínico precisa adequar-se de maneira a impedir a influência de fatores que levem a distorções na temperatura ambiente. No ambiente clínico, em meio a várias especialidades, a termografia se apresenta como ferramenta promissora no auxílio do diagnóstico e mapeamento da dor para o planejamento do tratamento de diversas patologias, como destacado nos estudos selecionados em diversas áreas (Melo et al.,2019; Zhou et al.,2020).

Segundo Endo et al.,2019, a termografia foi útil para a avaliação quantitativa da temperatura facial em pacientes submetidos a cirurgia ortognática, no qual identificaram em seu estudo as mudanças de temperatura no pós-operatório, que foram predominantemente ligadas a inflamação após a cirurgia.

Sobre as Disfunções temporomandibulares (DTMs), Rytivaara et al.,2021, apontaram que a termografia pode ser uma ferramenta potencial para o diagnóstico de DTM em pacientes do sexo femininos, sugerindo em seus resultados que as informações térmicas avaliadas em áreas faciais específicas podem ajudar a descriminar pacientes com DTM de pacientes sem DTM, podendo também ser utilizada para quantificar a dor associada.

Em relação à hiperatividade muscular, que pode desencadear aumento da pressão intra-articular no funcionamento da ATM, Clemente et al.,2018, investigaram o tratamento adequado a uma instrumentalista de sopro com DTM, onde foi utilizado como ferramenta complementar de diagnóstico a termografia, que se mostrou fundamental para compreender a biomecânica da articulação temporomandibular, contribuindo com um diagnóstico mais rápido e correto.

Entretanto, Barbosa et al. 2020, e Melo et al. 2019, destacaram em seus estudos a baixa acurácia e a escassez na literatura quanto à confiabilidade do uso da termografia na DTM, respectivamente. Barbosa et al.2020, identificaram também que pacientes com DTM não apresentam diferenças assimétricas de temperatura nas regiões de interesses analisadas e que a intensidade da dor à palpação em pacientes com DTM pode ser acompanhada por uma diminuição da temperatura local de acordo com os parâmetros térmicos.

Altindiş e Güngörmüş 2019, levaram os dados termográficos em consideração para identificar que as modalidades de tratamento com placa oclusal e terapia a laser de baixa potência (LLLT) foram consideradas eficazes no tratamento da síndrome da dor miofascial. Após os exames térmicos afirmaram que o tratamento com LLLT poderiam fornecer resultados mais vantajosos nesses pacientes e que avaliações mais objetivas poderiam ser feitas utilizando a termografia infravermelha. 
A termografia infravermelha pode ser aplicada também em estudos laboratoriais, desde que exista também uma padronização na temperatura ambiente onde o exame será aplicado, em diversas áreas como na Endodontia (Diegritz et al.,2020), Ortodontia (Lipski et al.,2020), Dentística (Luximon et al.,2016) e Implantodontia (Möhlhenrich et al.,2016; Scarano et al.,2020).

No estudo in vitro de LipskiI et al. 2020, os autores utilizaram como padronização uma câmera térmica e seu pacote de software para fabricação de coroa em resina. Para detectar a mudança de temperatura, a câmera foi montada em um pedestal perpendicular ao lado interno do teto da câmara a uma distância de $15 \mathrm{~cm}$. Os termogramas foram registrados em intervalos de 2 segundos durante um período de 6 minutos, em um ambiente com temperatura controlada (temperatura ambiente em $20 \pm 1,8$ ${ }^{\circ} \mathrm{C}$, umidade $50 \pm 5 \%$ e fluxo de ar menor que $0,5 \mathrm{~m} / \mathrm{s}$ ). As câmeras foram calibradas para distância, temperatura ambiental e emissividade do tecido radicular.

Já Kurt et al. 2017, utilizaram a câmera térmica e seu próprio pacote de software, para medir as mudanças de temperatura durante diferentes procedimentos de limpeza. As amostras foram colocadas em blocos de acrílico fixados perpendicularmente em uma placa especialmente projetada, posicionadas a uma distância de $15 \mathrm{~cm}$ do local pulpar das amostras para padronizar as medidas térmicas e registros da câmera para todos os espécimes. A câmera possuía resolução 320 x 240 pixels, campo de visão $25 \times 19^{\circ}$, e a janela de temperatura foi ajustada para um mínimo de $20^{\circ} \mathrm{C}$ e uma máximo de $40^{\circ} \mathrm{C}$. Todos os registros foram realizados em uma sala especial com condições ambientais controladas (Temperatura ambiente em 24 $\pm 0,9^{\circ} \mathrm{C}$; umidade relativa em $44 \pm 5 \%$; fluxo de ar em $0,5 \mathrm{~m} / \mathrm{s}$ ).

Diegritz et al. 2020, utilizaram uma câmera térmica com resolução de 382 x 288 pixels e faixa de temperatura de -20 a $900^{\circ} \mathrm{C}$. o campo de medição foi focado na superfície da raiz. A emissividade foi calibrada com um termopar (PL - $120 \mathrm{~T} 1$, Voltcraft, Wollerau, Sueça) e ajustada para 0,900. Para uma padronização, os dentes foram colocados em uma caixa de acrílico especialmente projetada durante o procedimento de obturação. A temperatura da caixa foi mantida em $37^{\circ} \mathrm{C}$ aguardando um período de equilíbrio antes do início da obturação. As mudanças da temperatura na superfície da raiz foram determinadas em 3 e em 1 min após a ativação do plugador de calor. Dessa forma, a termografia pode ser bem aplicada em estudos laboratoriais na Endodontia.

Na implantodontia, o estudo de Scarano, Lorusso e Noumbis 2020, utilizou soro fisiológico para simular a temperatura corporal $\left(37^{\circ} \mathrm{C}\right)$, onde costelas bovinas foram então presas a uma placa de base de alumínio, e o preparo para colocação dos implantes começou quando a temperatura interna do osso atingiu a temperatura de $37 \pm 0,1^{\circ} \mathrm{C}$. As medidas térmicas foram realizadas em uma sala climatizada com temperatura entre 23 e $24^{\circ} \mathrm{C}$, umidade relativa $50 \pm 5 \%$ e sem ventilação direta do osso. Já Möhlhenrich et al. 2016, utilizaram a termografia também em Implantodontia, realizando as imagens com uma câmera térmica digital de 14 bits (FLIR17 PRICE BURNER, Flir Systems, Danderyd, Suécia) com uma matriz de plano focal 320x240, faixa espacial de $9 \mathrm{~mm}$, realizando o posicionamento da câmera a $50 \mathrm{~cm}$ de distância do bloco ósseo de teste para obter uma resolução máxima.

Dos estudos clínicos selecionados, os protocolos adotados foram melhores descritos em Rytivaara et al. 2021, que dispôs para o exame uma sala sem janelas, com temperatura estabilizada em $22^{\circ} \mathrm{C}$, condições essa em que, anteriormente a aquisição das termografias, os participantes permaneceram em repouso por 30 minutos para estabilizar a temperatura corporal, onde foram levados em consideração aspectos fisiológicos dos participantes como índice de massa corpórea (IMC), como também foram considerados inclusive aspectos fisiológicos, como quanto ao período menstrual das participantes do sexo feminino, estes que poderiam trazer incongruências aos resultados. Neste mesmo estudo foi utilizado um apoio de mento para manter estático o paciente e proporcionar uma melhor exposição à região de interesse. E a fim de evitar reflexos no termograma foi disposto um fundo de espuma preta posterior ao paciente. 
Já no estudo de Barbosa et al. 2020, os participantes foram instruídos a não utilizar cosméticos na face e não se expor a fontes de calor, não fazer uso de alguns medicamentos, não praticar exercícios físicos e não fazer contato com a pele de modo a gerar calor facial. Tendo sido a sala preparada com ar-condicionado com temperatura de $23^{\circ} \mathrm{C}$, umidade relativa entre $40 \%$ e $60 \%$, verificada por termohigrômetro digital, as paredes foram revestidas com folhas de poliestireno expandido (isopor), folhas de alumínio e emborrachado preto. Também com o objetivo de evitar interferência por fontes externas de calor, além disso apenas 2 examinadores e o voluntário permaneceram na sala durante o exame.

Além das aplicações relatadas, também é possível verificar estudos que objetivaram analisar o bem-estar dos profissionais de saúde, conforme foram abordados em Scarano, Inchingolo e Lorusso 2020, que através de imagens termográficas identificaram que as máscaras N95, comumente utilizadas no âmbito da pandemia, causam um aumento significativo na temperatura facial, trazendo com seu uso um desconforto e diurese respiratória, até mesmo logo após seu uso. Neste caso, com o objetivo de analisar cirurgiões-dentistas, Cosoroaba et al. 2019, aplicaram a termografia voltada desta vez para a ergonomia destes profissionais, identificando determinadas posições de estiramento muscular, exercidas durante suas rotinas de trabalho. Segundo os autores, tais posições podem desencadear distúrbios musculoesqueléticos (DME), principalmente ocasionadas por posturas incorretas, ocorrendo uma elevação de temperatura nas regiões anatômicas acometidas, podendo ser identificada nos termogramas.

A escassez de artigos relevantes encontrados sobre a aplicabilidade da termografia na Odontologia foi uma das limitações desta pesquisa. Sugere-se a realização de mais estudos clínicos, bem como laboratoriais, utilizando a termografia infravermelha como método coadjuvante tanto no diagnóstico, quanto no acompanhamento de terapias.

\section{Considerações Finais}

Com base na pesquisa realizada, verifica-se que ainda não existe uma quantidade significativa de artigos que abordem as aplicações da termografia na Odontologia, havendo ainda diversas áreas que podem utilizá-la como ferramenta de diagnóstico e investigação eficaz. Por ser uma técnica não invasiva e de baixo custo, demonstra-se ser um método promissor na área da odontologia, destacando-se como exame complementar durante diagnóstico e tratamento de DTM e em exames laboratoriais com a Implantodontia.

Considerando os parâmetros adotados pelos estudos clínicos abordados na realização dos exames termográficos, houve uma semelhança entre eles, relacionados ao ambiente do exame quanto à temperatura, iluminação, umidade, distância do equipamento ao paciente. Porém, não foi seguido protocolo ou regra única, havendo diferenças em diversos aspectos, onde, estas, mesmo sendo mínimas, podem trazer incongruências aos exames. Devendo ainda considerar os diferentes equipamentos utilizados, softwares de tratamento de imagens. Diante do exposto, fica evidenciado o fato de que a não existência de um protocolo específico a ser adotado pode trazer de um mesmo tipo de exame diversos resultados.

Por trata-se de um tema relativamente novo, os autores sugerem que sejam feitos novos estudos utilizando de novas ferramentas, que venham a surgir com o avanço da tecnologia.

\section{Referências}

Altindiş, T., \& Güngörmüş, M. (2019). Thermographic evaluation of occlusal splint and low-level laser therapy in myofascial pain syndrome. Complement Ther Med. Jun, 44:277-281. 10.1016/j.ctim.2019.05.006.

Amorim, A. M. A. M., Barbosa, J. S., Freitas, A. P. L. F., Viana, J. E., Vieira, L. E. M., Suassuna, F. C. M., Bento, P. M. \& Melo, D. P. (2018). Termografia infravermelha na Odontologia. HU Revista, Juiz de Fora, 44(1): 15-22.

Barbosa, J. S., Amorim, A, Arruda, M., Medeiros, G., Freitas, A., Vieira, L., Melo, D. P., \& Bento, P. M. (2020). Infrared thermography assessment of patients with temporomandibular disorders. Dentomaxillofac Radiol. May 1,49(4):20190392. 10.1259/dmfr.20190392. 
Behnia, A. \& Mcdonald, N. J. (2001). Avaliação termográfica infravermelha in vitro da raiz temperaturas da superfície geradas pelo sistema thermafil plus. Journal of Endodontics, 93(3), 203-5.

Brioschi, M. L, Teixeira M. J., Silva, F. M., \& Colman, D. (2010). Princípios e Indicações da Termografia Médica. Andreoli.

Brioschi, M. L. (2011). Metodologia de normalização de análise do campo de temperaturas em imagem infravermelha humana. 110f. 2011. Tese (Doutorado em Engenharia Mecânica) - Universidade Federal do Paraná, Curitiba.

Brioschi, M. L., Macedo, J. F. \& Macedo, R. A. C. (2001). Termometria Cutânea Infravermelha de Alta Sensibilidade (T.I.A.S.) - Definição, Aplicações e Especificações. RevMed Paraná, 59: 56-63.

Chakrabort Y. M. (2016). A new approach of oral cancer detection using bilateral texture features in digital infrared thermal images. Conference proceedings. IEEE Engineering in Medicine and Biology Society Soc., 1377-1380.

Christensen. J., Vaeth M. \& Wenzel A. (2012). Thermographicimagingoffacialskin-genderdifferencesandtemperaturechanges over time in healthy subjects. Dento maxillo facial Radiology. 41, 662-7.

Clemente M. P., Mendes, J., Moreira, A., Vardasca, R., Ferreira, A. P., \& Amarante, J. M. (2018). Wind Instrumentalists and Temporomandibular Disorder: From Diagnosis to Treatment. Dent J (Basel). 6(3):41. 10.3390/dj6030041.

Cosoroaba, M. R, Cirin, L., Anghel, M. D., Talpos-Niculescu, C. I., Argesanu, V., Farkas, A. Z., \& Negrutiu, M. L. (2019). The use of thermal imaging in evaluating musculoskeletal disorders in dentists. J Med Life. 12(3):247-252. 10.25122/jml-2019-0017.

Melo, D. P, Bento, P. M., Peixoto, L. R., Martins, S. K. L. D., \& Martins, C. C. (2019). Is infrared thermography effective in the diagnosis of temporomandibular disorders? A systematic review. Oral Surg Oral Med Oral Pathol Oral Radiol. 127(2):185-192. 10.1016/j.00oo.2018.09.006

Diegritz C, Gerlitzki O, Fotiadou C \& Folwaczny M. (2020). Temperature changes on the root surface during application of warm vertical compaction using three different obturation units. Odontology. 108(3):358-365. 10.1007/s10266-019-00472-0.

Endo T, Komatsuzaki A, Miyagawa Y, Kamoda T, Goto S, Koide K \& Mizutani M. (2019). Thermographic assessment of facial temperature in patients undergoing orthognathic surgery. J Oral Sci. 61(2):321-326. 10.2334/josnusd.18-0194.

Fricova, J., Janatova, M., Anders, M., Albrecht, J. \& Rokyta, R. (2018). Thermo vision: a new diagnostic method for orofacial pain? J Pain Res., 13, 31953203 .

Haddad, D. S., Brioschi, M. L. \& Arita, E. S. (2012). Thermographic and clinical correlation of myofascial trigger points in the masticatory muscles. Dento maxilla fac Radiol. 41, 621-9.

Haddad, D.S. et al. (2014). Thermographic characterization of masticatory muscle regions in volunteers with and without myogenous temporomandibular disorder: preliminary results. Dentomaxillofacial Radiology, $43, \mathrm{n} .8$.

Hakguder, A. et al. (2003). Efficacy of low-level laser therapy in myofascial pain syndrome: an algometric and thermographic evaluation. Lasers in Surgery and Medicine, 33, 339-43.

Harder, S. et al. (2018). Intraosseous Temperature Changes During Implant Site Preparation: In Vitro Comparison of Thermo couples and Infrared Thermography. The International Journal of Ora I\& Maxillo facial Implants., 33, 72-78.

Iosif, L. et al. (2016). Clinical study on thermography, as modern investigation method for Candida-associated denture estomatitis. Romanian Journal of Morphologyand Embryology., 57, 191-5

Kurt, G., Gül, N., Er, Ö., Çakmak, G., Bendeş, E.,, \& Aslantaş, V. (2017). Thermal imaging of the pulp during residual adhesive removal. J Orofac Orthop. 78(4):330-337. English. 10.1007/s00056-017-0089-x.

Koche, J. C. (2011). Fundamentos de metodologia científica. Petrópolis: Vozes. http://www.brunovivas.com/wp content/uploads/sites/10/201 8/07/K\%C3\%B6che-Jos\%C3\%A9-Carlos0D0AFundamentos-de-metodologia-cient\%C3\%ADfica-_teoria-da0D0Aci\%C3\%AAncia-e-inicia\%C3\%A7\%C3\% A3o -\%C3\%A0pesquisa. pdfhttps://repositorio.ufsm.br/bitstream/handle/1/15824/Lic_Computacao_MetodologiaPesquisaCientifica.pdf?sequ ence=1ream/han dle /1/15824/Lic_Computacao_Metodologia Pesquisa-Cientifica.pdf?sequence=1

Lipski, M., Woźniak, K., Szyszka-Sommerfeld, L., Borawski M, Droździk, A., \& Nowicka, A. (2020). In Vitro Infrared Thermographic Assessment of Temperature Change, in the Pulp Chamber during Provisionalization: Effect of Remaining Dentin Thickness. J Healthc Eng. 10.1155/2020/8838329. PMID: 33224457, PMCID: PMC7671814.

Luximon Y, Anne Sheen K \& Luximon A. (2016). Time dependent infrared thermographic evaluation of facemasks. Work. 54(4):825-35. 10.3233/WOR162353.

Marroquín, B. et al. (2015). Thermo plastic properties of endodontic gutta-percha: a thermographic in vitro study. Journal of Endodontics, 41, 79-82

Möhlhenrich S.C, Abouridouane M, Heussen N, Modabber A, Klocke F \& Hölzle F. (2016). Influence of bone density and implant drill diameter on the resulting axial force and temperature development in implant burs and artificial bone: an in vitro study. Oral Maxillofac Surg. 20(2):135-42. 10.1007/s10006015-0536-z.

Rytivaara R, Näpänkangas R, Kainulainen T, Sipola A, Kallio-Pulkkinen S, Raustia, A. \& Thevenot J. (2021). Thermographic findings related to facial pain a survey of 40 subjects. Cranio. 1-8. 10.1080/08869634.2021.1894859.

Scarano A, Inchingolo F \& Lorusso F. (2020). Facial Skin Temperature and Discomfort When Wearing Protective Face Masks: Thermal Infrared Imaging Evaluation and Hands Moving the Mask. Int J Environ Res Public Health. 17(13):4624. 10.3390/ijerph17134624. 
Research, Society and Development, v. 10, n. 8, e40210817479, 2021

(CC BY 4.0) | ISSN 2525-3409 | DOI: http://dx.doi.org/10.33448/rsd-v10i8.17479

Scarano A, Lorusso F \& Noumbissi S. (2020). Infrared Thermographic Evaluation of Temperature Modifications Induced during Implant Site Preparation with Steel vs. Zirconia Implant Drill. J Clin Med. 9(1):148. 10.3390/jcm9010148.

Zhou, Y., Ghassemi, P., Chen, M., Mcbride, D., Casamento, J.P., Pfefer, T.J. \& Wang, Q. (2020). Clinical evaluation of fever-screening thermography: impact of consensus guideline sand facial measure men location. J BiomedOpt., 25(9). 